Les premiers résultats de la mission sont très encourageants et montrent clairement que les petits satellites représentent une méthode rapide pour répondre à des questions scientifiques clés. À l'ère du NewSpace, c'est-à-dire une ère bénéficiant des progrès technologiques de la miniaturisation et de coûts raisonnables d'accès à l'espace, cette mission montre clairement qu'une constellation de petits satellites dédiés à la mesure du déséquilibre énergétique de la Terre est envisageable.

Malgré l'urgence climatique, il reste difficile d'obtenir des financements pour les missions satellitaires. Les nanosatellites offrent donc une opportunité à moindre coût pour la mise en œuvre de constellations. Ces missions doivent s'appuyer sur la mise en œuvre de constellations de satellites afin d'assurer une couverture spatiale et temporelle suffisante. Les constellations de satellites dédiées à l'étude du climat et à la science représentent aujourd'hui une évidence (Baker et al., 2020). Le prototype UVSQ-SAT ouvre la voie à une potentielle constellation Terra- $F$ composée de 50 petits satellites ayant les performances nécessaires pour répondre au besoin scientifique.

D'autres solutions pour mesurer le déséquilibre énergétique de la Terre sont possibles en utilisant deux satellites traditionnels placés aux points de Lagrange L1 et L2 afin d'effectuer des mesures des portions éclairées et non éclairées de notre planète. La principale ambition est de réaliser des mesures du déséquilibre énergétique de la Terre au sommet de l'atmosphère et de sa variabilité dans le temps afin de détecter toute tendance globale à long terme avec une stabilité d'au moins $\pm 0,2 \mathrm{~W} \mathrm{~m}^{-2}$ par décennie, mais aussi de pouvoir obtenir des résolutions spatiales de quelques dizaines de kilomètres et temporelles de trois heures avec une précision de quelques watts par mètre carré.

UVSQ-SAT et sa potentielle future constellation abordent la même thématique scientifique que le programme Forum (Far-infrared Outgoing Radiation Understanding and Monitoring) du programme Earth Explorer de l'Esa, mais sous un angle complètement différent.

Mustapha Meftah, Philippe Keckhut Latmos, CNRS / UVSQ / Université Paris-Saclay/Sorbonne Université

\footnotetext{
Pour en savoir plus

http://uvsq-sat.projet.latmos.ipsl.fr

Baker D., Chandran A., Chang L., Macdonald M., Meftah M., Millan R., Park J. H., Kumar P., Price C., von Steiger R., Wu J. et al., 2020. An international constellation of small spacecraft. Space Research Today, 208, 23-28.

Gristey J.J., Chiu J.C., Gurney R.J., Han S.C., Morcrette C.J., 2017. Determination of global Earth outgoing radiation at high temporal resolution using a theoretical constellation of satellites. J. Geophys. Res., 122, 1114-1131.

Kiehl J.T., Trenberth K.E., 1997. Earth's annual global mean energy budget. Bull. Am. Meteorol. Soc., 78, $197-197$.

Meftah M., Damé L., Keckhut P., Bekki S. et al., 2020. UVSQ-SAT, a pathfinder CubeSat mission for observing essential climate variables. Remote Sens., 12, 92. doi: 10.3390/rs12010092

Stephens G.L., Li J., Wild M., Clayson C.A., Loeb N., Kato S., L'Ecuyer T., Stackhouse P.W., Lebsock M., Andrews T., 2012. An update on Earth's energy balance in light of the latest global observations. Nat. Geosci., 5, 691-696.

Twiggs R.J., 2000. Space system developments at Stanford University: From launch experience of microsatellites to the proposed future use of picosatellites. SPIE 4136. Small Payloads in Space, 4136, 79-86. doi: 10.1117/12.406646

von Schuckmann K., Cheng L., Palmer M.D. et al., 2020. Heat stored in the Earth system: Where does the energy go? Earth Syst. Sci. Data, $12,2013-2041$.
}

\title{
Arktika-M1, un satellite météorologique russe en orbite excentrée
}

Les orbites des satellites météorologiques ont été largement évoquées dans cette revue (Capderou, 2017a, b). L'actualité - le lancement du satellite météorologique russe Arktika-M1, le premier du programme Arktika-M - nous incite à revenir sur le sujet, car l'orbite de cette famille de satellites est très originale pour ce type de mission.

Les (magnifiques) images de Météosat ont vulgarisé, pratiquement banalisé, le concept de satellite géostationnaire. Tout honnête homme sait à présent que l'orbite de ces satellites est équatoriale et circulaire. Leur altitude est très élevée : 5,61 rayons terrestres, soit environ $36000 \mathrm{~km}$. Et on constate vite, en observant les images (un disque représentant presque la moitié du globe terrestre) que les régions près des pôles sont absentes ou très mal représentées.

Tableau 1. Caractéristiques orbitales pour deux types de satellites (voir Capderou, 2017a) : a le demigrand axe, e l'excentricité (sans dimension), $i$ l'inclinaison, $h_{a}$ l'altitude à l'apogée et $h_{p}$ l'altitude au périgée.

\begin{tabular}{lccccc} 
& $\boldsymbol{a}(\mathbf{k m})$ & $\boldsymbol{e}$ & $\boldsymbol{i}\left({ }^{\circ}\right)$ & $\boldsymbol{h}_{\mathrm{a}}(\mathbf{k m})$ & $\boldsymbol{h}_{\mathrm{p}}(\mathbf{k m})$ \\
Molnya classique & 26552,8 & 0,75 & 63,4 & 40089 & 260 \\
\hline Arktika-M1 & 26552,8 & 0,72 & 63,3 & 39310 & 1073 \\
\hline
\end{tabular}

On admet qu'au-delà de $55^{\circ}$ de latitude nord les images ne sont pas correctement exploitables. En ce qui concerne l'Europe, un satellite géostationnaire ne présente pas d'intérêt pour l'Islande, l'Écosse, les pays scandinaves et la Russie.

Ce fait, les Russes l'ont intégré depuis longtemps, dès le début de l'ère spatiale, qui avait si brillamment commencé pour l'URSS. Pour contourner ces problèmes de position géographique intangible, les ingénieurs soviétiques ont inventé l'orbite Molnya. Cette orbite est une ellipse très excentrée dont les caractéristiques sont notées dans le tableau 1. La période est proche de 12 heures; plus exactement, elle est égale à un demi-jour sidéral, soit 717,72 minutes. Une autre propriété de cette orbite est qu'elle est " phasée » (Capderou 2012, 2014), c'est-à-dire que sa trace repasse perpétuellement sur elle-même, jour après jour.

L'application de la deuxième loi de Kepler montre que le satellite se déplace très lentement près de l'apogée et très rapidement près du périgée ; 


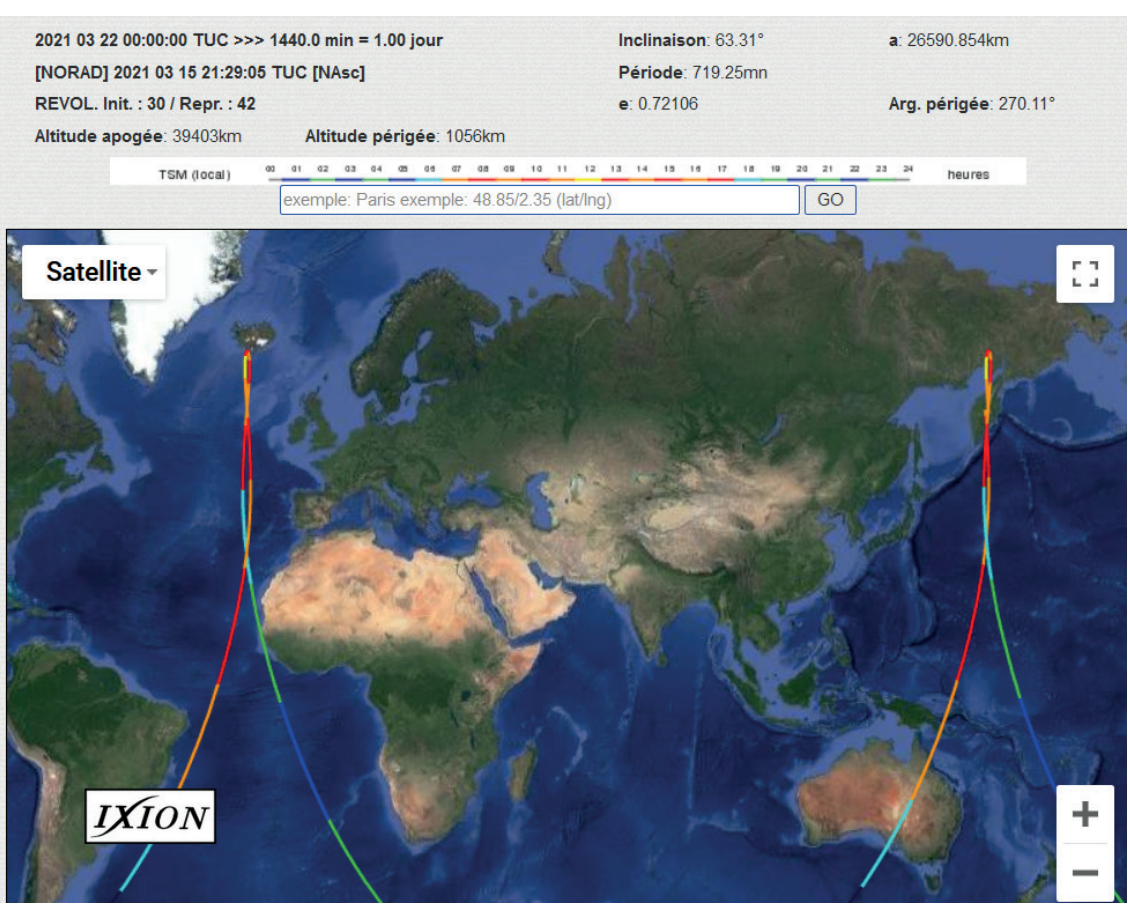

Figure 1. Trace de l'orbite du satellite Arktika-M1 avec notation du TSM (temps solaire moyen : heure locale).

le rapport entre ces deux vitesses extrêmes est d'autant plus important que l'excentricité est grande. L'orbite Molnya a été calculée pour que, lorsque le satellite est dans les environs de l'apogée, la vitesse de sa trace au sol soit proche de celle de la Terre à ces latitudes. Le satellite, pendant tout un intervalle de temps (plusieurs heures, comme nous verrons), peut être considéré comme géostationnaire.

Mais un autre point important intervient ici. Sous l'effet des perturbations (principalement celles dues à la forme ellipsoïdale de la Terre), le périgée (et donc l'apogée) ne reste pas en place sur son orbite. Il se déplace, il « circule » sur l'orbite, dans un mouvement fondamental en mécanique spatiale appelé précession apsidale. Cette vitesse de précession dépend de divers

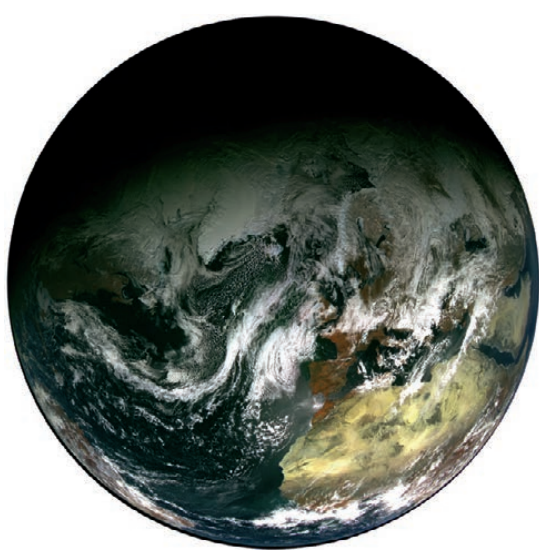

Figure 2. Première image diffusée obtenue par le satellite russe Arktika-M1. Image du 22 mars 2021, 12:37 TU (15:37 à Moscou). que l'inclinaison soit de $63^{\circ}$ (ou de son facteurs (caractéristiques orbitales du satellite et données géodésiques de la planète-corps attractif), mais les équations montrent qu'on peut l'annuler. Pour cela, une condition et une seule : supplément, $117^{\circ}$ ).

Autant l'empire russe ne pouvait profiter des orbites géostationnaires, autant il est favorisé par les dieux de la mécanique céleste. Cette inclinaison de $63^{\circ}$ (cette valeur ne dépend d'aucune grandeur orbitale, géodésique, elle résulte d'un simple arc cosinus) intéresse au plus haut point la Russie qui étend son territoire sur une dizaine de fuseaux

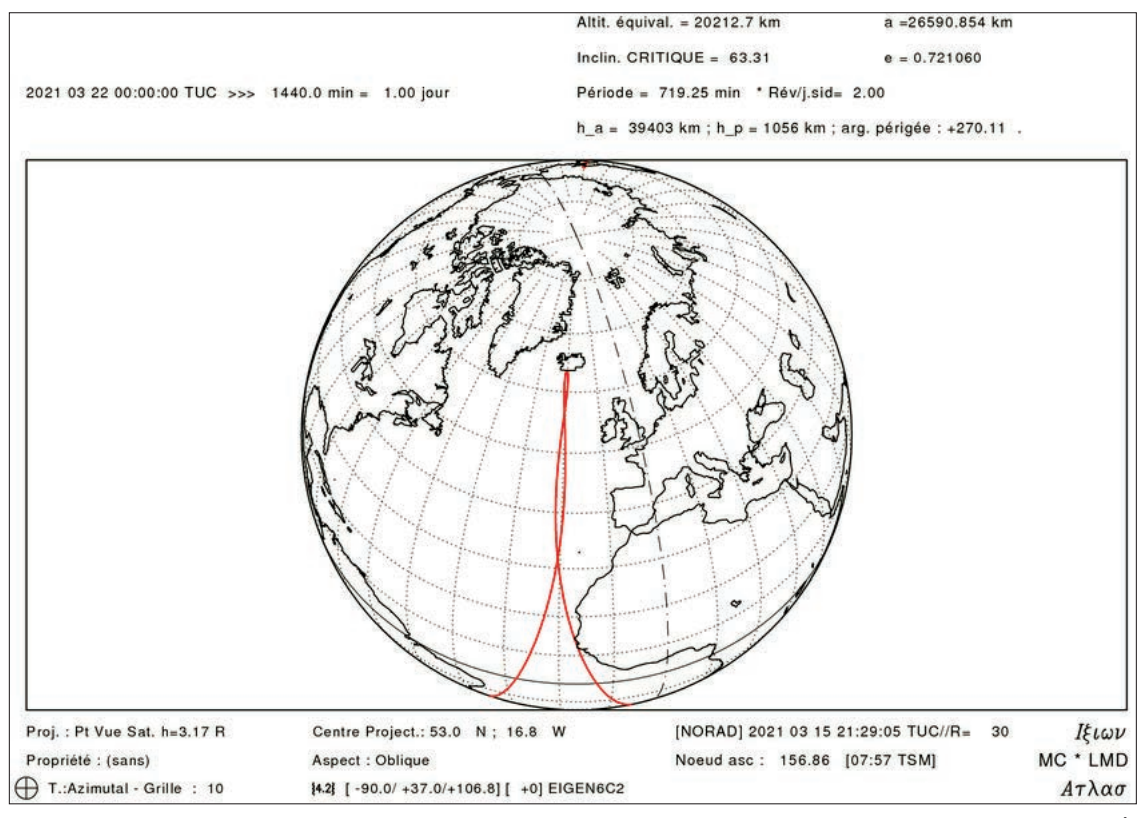

Figure 3. Reconstitution de la prise de vue du satellite Arktika-M1, le 22 mars 2021, 12:37 TU. À comparer avec la figure 2 .

Période $=719.25 \mathrm{~min} \cdot$ Rév $/ \mathrm{j}$.sid $=2.00$

$\mathrm{h} \_\mathrm{a}=39403 \mathrm{~km} ; \mathrm{h} \_\mathrm{p}=1056 \mathrm{~km}$; arg. périgée : +270.11 horaires en longitude, de part et d'autre de la latitude de $63^{\circ} \mathrm{N}$.

À partir de 1964 (Spoutnik-1 date de 1957), les satellites Molnya ont été lancés à un rythme soutenu, surtout pendant la guerre froide. En comptabilisant les trois séries Molnya-1, 2 et 3, on arrive à près de deux cents satellites. C'étaient officiellement des satellites de communication interne à l'URSS, puis à la Russie. Vu le nombre, il devait bien y avoir d'autres utilisations, plus secrètes.

La Russie s'est équipée depuis longtemps de satellites météorologiques classiques (Capderou, 2017b). Mais c'est la première fois, avec Arktika-M, que l'orbite Molnya, pourtant surexploitée depuis plus de 50 ans, est utilisée pour ce type de mission.

Observons plus en détail, figure 1, cette orbite Molnya dans le cas d'Arktika-M1.

Les calculs montrent (Capderou, 2012, 2014) que, sur une révolution de 12 heures, le satellite passe 11 heures dans l'hémisphère Nord et 1 heure dans l'hémisphère Sud. Le temps passé dans la partie « bouclée » de la trace est de 10 heures, durée pendant laquelle on peut considérer le satellite comme stationnaire. Son altitude varie, mais le point subsatellite est pratiquement fixe.

Sur la figure 1, les heures locales (TSM) ont été notées par des couleurs. Sur la trace ayant l'apogée sur l'Islande, on note le défilement de dix heures locales environ, entre 8 et $18 \mathrm{~h}$ TSM. Douze heures plus tard, lors de la

TSM. Douze heures plus tard, lors de la A 
révolution suivante, on retrouve une situation identique à l'extrémité orientale de la Sibérie.

Cette orbite permet à Arktika-M1 de réaliser ses observations pendant deux périodes de dix heures, chacune étant centrée sur midi solaire (12 h TSM). Cela donne donc 20 heures d'observation dans de bonnes conditions sur une journée.

Le service météorologique russe a diffusé la première image obtenue par
Arktika-M1 (figure 2). Elle donne la même impression qu'une vue de satellite géostationnaire, l'altitude est à peu près la même.

En connaissant l'instant précis de la prise de vue, le logiciel Ixion calcule la position et l'altitude du satellite à partir des données Norad (Capderou, 2017a). On peut restituer les conditions exactes de vue (figure 3 ).

L'intérêt de l'orbite Arktika apparaît clairement sur les figures 1, 2 et 3 . La vue sur la zone polaire nord est quasi permanente (20 heures sur 24). La même orbite exactement, avec seulement un changement de l'argument du périgée $\left(90^{\circ}\right.$ au lieu de $\left.270^{\circ}\right)$, permettrait d'avoir le même point de vue sur la zone polaire sud. Mais qui va financer Antarktika? Laboratoire de météorologie dynamique, Sorbonne Université / CNRS / École polytechnique, Palaiseau

\section{Ouranos AuRA : accompagner les territoires dans leur transition climatique}

La structure Ouranos AuRA, collectif de chercheurs de la région AuvergneRhône-Alpes principalement centré sur le pôle universitaire grenoblois, a pour objectif de faire le lien entre science et société sur la question du changement climatique et de ses impacts sur les territoires, particulièrement en montagne. Les collectivités des Alpes du Nord sont en effet particulièrement impactées par les premiers effets du réchauffement dans leurs activités agricoles ou forestières et dans l'industrie des sports de montagne et du tourisme, du fait, entre autres, d'un moindre enneigement et du recul glaciaire. Elles doivent aussi faire face à une recrudescence de phénomènes naturels extrêmes qui viennent régulièrement perturber les réseaux de transport ou parfois même frapper les populations. Les acteurs de ces collectivités sont incités par la réglementation à prendre en charge ces effets et à mettre en place des mesures pour y faire face localement, tant pour réduire les causes du réchauffement en jouant sur des mesures d'atténuation qu'en affrontant les effets immédiats au travers des stratégies d'adaptation. Cependant, si les outils de planification existent (les Plans Climat Air Énergie Territoriaux, PCAET, par exemple), ils restent souvent assez flous sur la mise en œuvre de mesures réellement concrètes sur le terrain. Des connaissances font défaut sur leurs répercussions à différentes échelles de temps et d'espace, alors qu'elles permettraient de mieux anticiper l'avenir des territoires. Mais, au-delà de ces connaissances, l'approche même des nouvelles formes de développement sont à réinventer dans un contexte de changement climatique et plus largement de changement global. Si les acteurs des territoires ont conscience de cette nécessité, les méthodes pour leurs mises en œuvre et pour accompagner ces changements leur font souvent défaut.

La communauté académique grenobloise est depuis plusieurs décennies investie dans l'étude du changement climatique et de ses impacts sur différents types de territoires et notamment sur les Alpes. Au-delà de l'implication de plusieurs scientifiques dans le Giec et l'IPBES, nombre d'entre eux, de différentes disciplines, sont également engagés dans des études plus locales, sur l'observation des évolutions climatiques, de leurs effets sur les glaciers, le pergélisol, les espèces végétales et animales, et les dynamiques territoriales. Ces travaux se font dans un cadre partenarial, plus ou moins soutenu, avec les acteurs locaux. Mais, devant les questions et défis qui se posent aujourd'hui pour les territoires, les demandes de collaboration se renforcent.

Ouranos AuRA a ainsi été créé en 2012 au sein du groupement d'intérêt scientifique (GIS) Envirhônalp, qui visait notamment à faciliter les interactions entre scientifiques et acteurs des territoires rhônalpins sur les questions environnementales au travers du développement de plateformes d'interface. Ouranos AuRA est donc l'une de ces plateformes, centrée sur le climat. Elle doit son nom au consortium Ouranos québécois, développé au début des années 2000, qui a vu dans notre demande une opportunité de déployer son modèle à l'international. L'idée de départ était d'une part de mettre à disposition les connaissances scientifiques développées dans les différents établissements académiques de la région Rhône-Alpes (devenue depuis Auvergne Rhône-Alpes) sur la question climatique et d'autre part d'identifier, dans les questions posées par les acteurs des territoires, les potentiels germes de problématiques de recherche à développer dans un partenariat science-société pour créer de nouvelles connaissances, utiles aux acteurs locaux. Depuis 2018 et la fin du GIS, les engagements d'Ouranos AuRA se poursuivent à l'échelle de l'ensemble de la nouvelle région Auvergne Rhône-Alpes, avec l'appui de la communauté académique du pôle grenoblois, très impliquée sur cette question, et de l'université GrenobleAlpes particulièrement. Plusieurs projets de recherche sont venus alimenter ces activités. Les derniers en date sont les projets Trajectories (financement 